\title{
Minimally Invasive Percutaneous Plate Osteosynthesis (Mippo) in Distal Tibial Fractures - A Prospective Study from Developing Nation
}

Naiyer Asif, Yasir Salam Siddiqui*, Jitesh Kumar Jain, Mohd Zahid, Mazhar Abbas, Aamir Bin Sabir and Sohail Ahmed

Department of Orthopaedic Surgery, J. N. Medical College, A.M.U., Aligarh, Uttar Pradesh, India

\begin{abstract}
Background: The management of distal tibial fractures can be challenging because of scarcity of soft tissues, their subcutaneous nature and poor vascularity. Consequently, operative treatment can lead to complications. The surgical treatment of fractures has evolved a great deal since the development of the original "open reduction and internal fixation" technique by the AO group. A mechanically stable fracture-bridging osteosynthesis can be obtained without significant dissection and surgical trauma to the bone and surrounding soft tissues by minimally invasive percutaneous plate osteosynthesis (MIPPO). This technique utilizes indirect reduction and internal fixation with locking compression plates. Our aim was to observe the outcome of the distal tibial fracture fixation (extra-articular and intra-articular) with locking compression plate (LCP) using MIPPO technique.
\end{abstract}

Material and Methods: 26 consecutive patients were prospectively underwent minimally invasive percutaneous plate osteosynthesis for distal tibial fractures between January 2010 and December 2011. Patients were aged between 20 to 65 years with seventeen males and nine females. Both open and closed AO types 43A, 43B and 43C fractures were included. Patients were managed with LCP using MIPPO technique. Postoperative regimen consisted of knee and ankle bending exercises starting on $1^{\text {st }}$ postoperative day. Non weight bearing ambulation started on $3^{\text {rd }}$ post-operative day, progressing to partial weight bearing at 6 to 8 weeks. Full weight bearing ambulation was allowed after clinico radiological union at 12 to 14 weeks.

Observation and Results: RTA was the commonest cause of injury. All fractures healed with good functional outcome. Mean union time was 4.5 months (range 3.5-8 months). Mean follow-up was 14.3 months. For all fractures acceptable active range of ankle movement was achieved. Mean AOFAS score was 88 . There were 2 cases of superficial infection, treated successfully with antibiotics. No deep infection, wound dehiscence and hardware failure was noted.

Conclusion: MIPPO is a well-documented technique that offers biological advantages and appears to be a reasonable treatment option for complex unstable distal tibial or pilon fractures that are not suited for intramedullary nailing. The literature is full of studies from developed nations, but studies from developing nations are lacking. Nevertheless small numbers of patients were involved in our study to draw any definitive conclusions, but our encouraging results will motivate the trauma surgeons from the developing nations to use MIPPO technique in managing distal tibial fractures, in an endeavor to reduce morbidity and associated complications, especially of the soft tissues.

Keywords: MIPPO technique; RTA; Fracture-bridging osteosynthesis; Locking compression plate; Indirect reduction

\section{Introduction}

Fractures of the distal tibial metaphysis with or without intraarticular extension can present a management challenge because of their inherent instability, scarcity of soft tissues, subcutaneous nature and poor vascularity of bone. Treatment modality is dictated by the fracture displacement, comminution, intra-articular extension and injury to the soft-tissue envelope [1]. The surgical treatment of fractures has evolved a great deal since the development of the original "open reduction and internal fixation" technique by the AO group, even though high rates of complications have been reported in literature with conventional methods [2-6]. Although intramedullary nailing minimizes surgical trauma to already traumatize soft tissues envelop, but is generally not considered suitable for distaltibial fractures because of lack of biomechanical stability of fixation and subsequent risk of malunion. Conventional plating results in extensive soft tissue dissection and periosteal injury, further compromising the blood supply to already traumatize soft tissues. External fixators are effective in managing compound fractures and fractures associated with extensive soft-tissue injury, but are also not free of complications, and are associated with a high incidence of pin tract infection, loosening and malunion [7]. A mechanically stable fracture-bridging osteosynthesis can be obtained without significant dissection and surgical trauma to the bone and surrounding soft tissues by minimally invasive percutaneous plate osteosynthesis (MIPPO) [8]. This technique utilizes indirect reduction and internal fixation with locking compression plates. The plate is tunneled subcutaneously, but extra periosteally, through limited skin incisions placed at the proximal and distal ends of the plate, bypassing the fracture site and then making a stable construct by judicious use of compression and locking screws. This technique aims to preserve bone biology and minimizes surgical trauma to already traumatized soft tissues. MIPPO with LCP offers biological advantages over conventional plating techniques in terms of low surgical trauma, preservation of the

*Corresponding author: Yasir Salam Siddiqui; Department of Orthopaedic Surgery, J. N. Medical College, A.M.U., Aligarh, Uttar Pradesh, India, Tel: +919837343400; E-mail: yassu98@gmail.com

Received January 25, 2014; Accepted May 28, 2014; Published May 30, 2014

Citation: Asif N, Siddiqui YS, Jain JK, Zahid M, Abbas M, et al. (2014) Minimally Invasive Percutaneous Plate Osteosynthesis (Mippo) in Distal Tibial Fractures - A Prospective Study from Developing Nation. J Trauma Treat S2: 009. doi:10.4172/2167-1222.S2-009

Copyright: (c) 2014 Asif N, et al. This is an open-access article distributed unde the terms of the Creative Commons Attribution License, which permits unrestricted use, distribution, and reproduction in any medium, provided the original author and source are credited. 
blood supply, lesser evacuation of osteogenic fracture hematoma and stable construct [8-10]. Our aim was to observe the results of the distal tibial fracture fixation with locking compression plate using MIPPO technique in terms of clinical and functional outcome.

\section{Material and Methods}

We prospectively followed the 26 consecutive patients who underwent minimally invasive percutaneous plate osteosynthesis using LCP for distal tibial fractures between January 2010 and December 2011. At start of study, permission was taken from hospital ethical committee and informed consent was obtained from all patients. Patients were aged between 20 to 65 years (mean age of 43 years) with seventeen males and nine females. We included all patients presenting to our outpatient or emergency department with high energy distal tibial fractures with or without intra-articular extension. All closed injuries, compound grade I and grade II fractures were included in the study. Compound grade III fractures were excluded from the study because of the presence of severe soft tissue injury, periosteal striping and loss of fracture hematoma, which may lead to confounding. There were 19 closed and 7 open fractures (compound grade $\mathrm{I}=5$ and grade $\mathrm{II}=2$ ). Antero-posterior and lateral radiographs of ankle including leg were used to determine the fracture personality (displacement, comminution, intra-articular extension) and then classifying the fracture accordingly (Figure 1). Fractures were classified according to the AO comprehensive classification system [11]. There were eighteen $43 \mathrm{~A}$, six $43 \mathrm{~B}$ and two $43 \mathrm{C}$ fractures. Open fractures were classified according to the Gustilo and Anderson classification system [12]. Fifteen patients were involved in road traffic accidents and eleven had a fall from height. All of the patients had an associated fibular fracture. On presentation all open fractures were managed with debridement and primary fixation, while closed injuries were managed initially with below knee plaster slab and elevation of the fractured extremity. The definitive fixation with locking compression plates using MIPPO technique was undertaken after subsidence of soft tissue trauma (Figure 2). Postoperative plaster cast immobilization was not used routinely. Postoperative regimen consisted of knee and ankle bending exercises starting on $1^{\text {st }}$ post-operative day. Non weight bearing ambulation started on $3^{\text {rd }}$ post-operative day, progressing to partial weight bearing at 6 to 8 weeks. Full weight bearing ambulation was allowed after clinico radiological union at 12 to 14 weeks. Patients were then followed up in the outpatient department at regular interval. At each visit patient were evaluated with clinical and radiological examination.

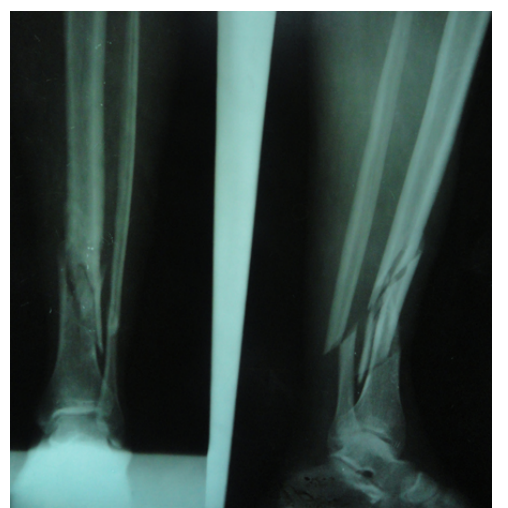

Figure 1: Antero-posterior and lateral radiograph of left ankle including leg showing comminuted, extra-articular fracture of lower end of tibia with fracture of lower third of fibula.

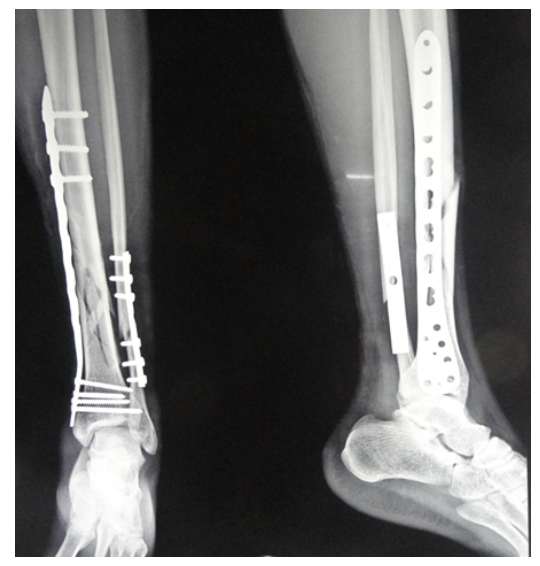

Figure 2: Immediate post-operative antero-posterior and lateral radiograph of left ankle including leg showing fixation of tibial fracture with LCP, and fibular fracture fixation with one third tubular plate. The radiograph shows satisfactory alignment.

The final outcome was evaluated at mean of 14.3 months. Clinical union was defined as single stance, painless full weight-bearing on the healed fractured extremity (Figure 3 ). Radiological union was defined as evidence of bridging callus in both the orthogonal views (Figure 4). Delayed union was labeled when patient does not met the above said criteria of union at nine months. Soft tissue outcome was evaluated from history of infection (superficial or deep), wound dehiscence, hardware impingement and necessity for hardware removal. American Orthopaedic Foot and Ankle Score (AOFAS), time back to work and change of work in relation to the injury were used to assess the functional outcome.

\section{Operative Technique}

The patient was positioned supine on operation table, with a thigh tourniquet. Intravenous antibiotics were given, before inflating tourniquet. Before proceeding to percutaneous plating, the fibular fracture was fixed first using a one-third tubular or recon plate, which helped in maintaining the length, alignment and indirect reduction of the tibial fracture (Figures $5 \mathrm{a}$ and $5 \mathrm{~b}$ ). In all cases fibular fixation was done. Following lateral malleolar fixation the integrity of syndesmosis was checked by pulling the distal fibula. After conventional plating of lateral malleolus, distal tibial LCP was tunneled sub-cutaneously, through limited skin incisions avoiding injury to the saphenous nerve and vein (Figure 5c). Plate was temporarily fixed using K-wires through the especially designed holes on the distal and proximal ends of the plate. Preliminary reduction and position of plate was checked under image intensifier. Interfragmentary compression was accomplished in selected cases with cortical screws placed through the plate (Figure $5 \mathrm{~d}$ ). Following inter fragmentary compression a mechanically stable construct was made by use of locking screws. All through the fixation care was taken to prevent posterior sagging of the tibia at the fracture site. Wound closure was done in layers without a suction drain. Following operation compression bandage was given, and limb was elevated. No splintage was used postoperatively.

\section{Observation and Results}

All patients were followed in outpatient department for clinical and radiological outcome. The mean number of days between admission and definitive fracture fixation was five days (range 0-9 days). Mean duration of operation was $95 \mathrm{~min}$ (range 80-125). The fibula was fixed 
Citation: Asif N, Siddiqui YS, Jain JK, Zahid M, Abbas M, et al. (2014) Minimally Invasive Percutaneous Plate Osteosynthesis (Mippo) in Distal Tibial Fractures - A Prospective Study from Developing Nation. J Trauma Treat S2: 009. doi:10.4172/2167-1222.S2-009

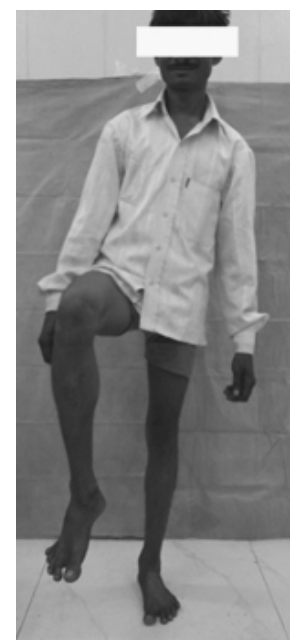

Figure 3: Clinical photograph of patient showing single stance, painless full weight-bearing on the healed fractured extremity - criteria defined for clinical union.

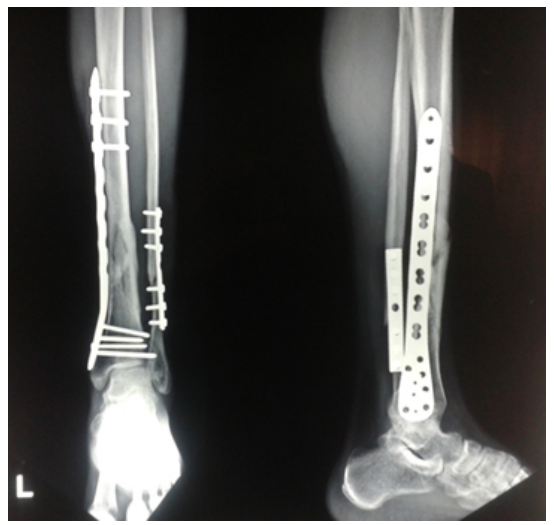

Figure 4: Post-operative antero-posterior and lateral radiograph of left ankle including leg showing union of both tibial and fibular fracture. The radiograph shows evidence of bridging callus in both the orthogonal views. Also note that no change in alignment is noted at fracture healing, indicating the effectiveness of fixation.

in all cases. Bone grafting was not done in any of our case. Mean followup was 14.3 months (9-23 months). Mean union time was4.5 months (range 3.5-8 months). Out of the 19 closed fractures, 15 fractures went to clinico radiological union within six months of operation, while rest of the four fractures took more than six months. Out of the 7open fractures, six fractures healed within six months, while one patient with compound grade II fracture required 10months for healing. There were two cases of superficial infection in open fracture group, managed successfully with antibiotics. None of the patient developed deep infection or wound dehiscence. All patients achieved functional active range of ankle movements. Mean AOFAS score was 88 at a mean of 14.3 months follow-up. There was no case of hardware failure, but four $(14.3 \%)$ patients reported plate impingement.

One patient in open fracture group developed delayed union. None of the patient developed non-union. Two patients developed 5 degrees of valgus mal-alignment, no significant recurvatum or pro-curvatum was noted in any of our case. No temporary fixation with an external fixator was done for initial soft tissue management. All patients were back to their pre-injury employment, and out of twenty six, 21 (80.8\%) patients were back to work within 6 months of injury, while rest of the patients required more than 6 months.

\section{Discussion}

The treatment of unstable distal tibial or pilon fractures remains controversial. Management and severity of the soft tissue injury is one of the most important determinants of the prognosis of distal tibial fractures. Complications such as wound dehiscence, infection, and non-union can be seen when soft tissue status is ignored [10]. Conventional methods of distal tibial fracture fixation have been associated with high rates of complications, such as wound infection, mal-union, non-union and hardware failure [2-6]. External fixation and intramedullary nailing techniques, beyond doubt minimize softtissue trauma and have been shown to diminish infection but their use is complicated by mal-union, non-union, unsatisfactory reduction of articular surface, and pin tract infection [2-6,13-15]

Open reduction in the distal tibia causes an increased risk of disrupting the blood supply, as shown by Borelli [16]. Hence, conventional plating of distal tibial fractures has been associated with

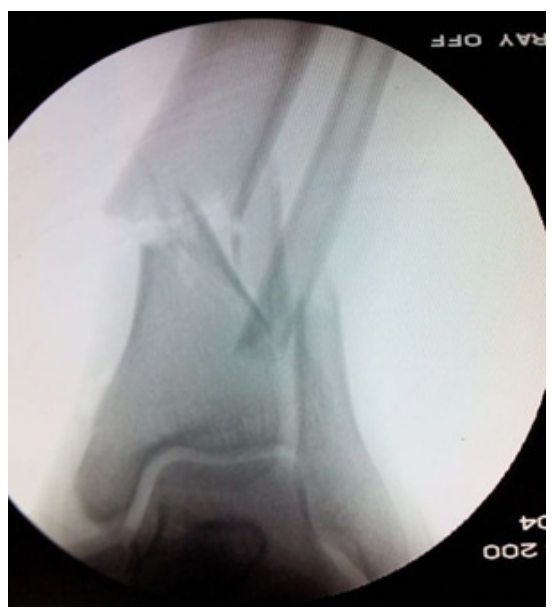

Figure 5a: Intra-operative fluoroscopic view showing displaced distal tibial and fibular fracture.

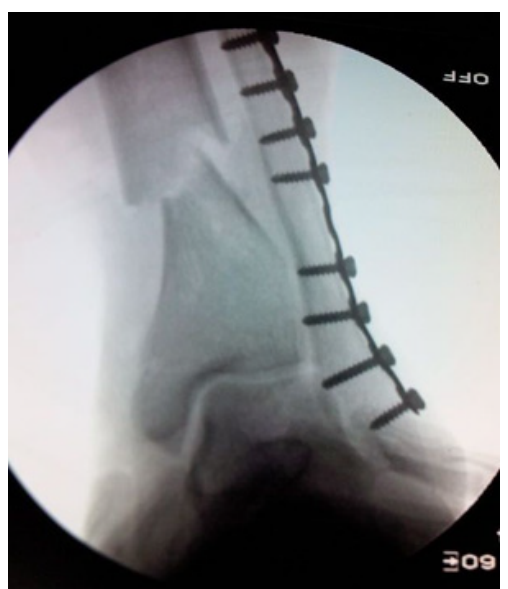

Figure 5b: Intra-operative fluoroscopic view showing maintenance of length, alignment and indirect reduction of the tibial fracture following fibular fracture fixation. 


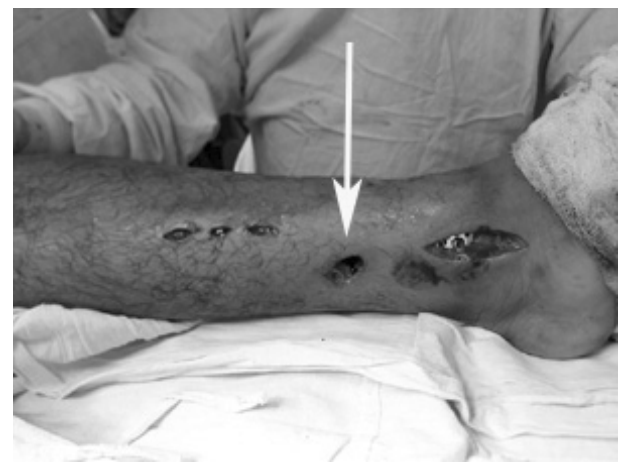

Figure 5c: Intra-operative photograph showing proximal and distal limited skin incisions. Distal incision is used to tunnel the LCP subcutaneously, while the proximal screws are for locking or cortical screws. Also note the compounding site (arrow head).

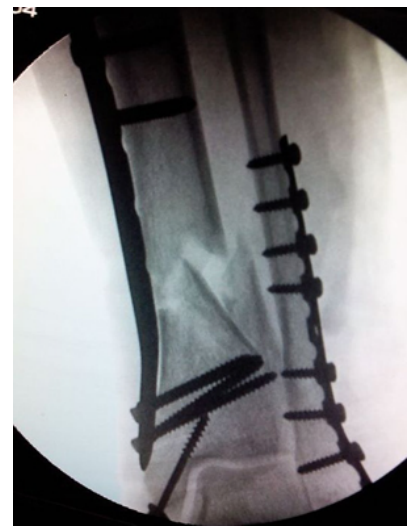

Figure 5d: Intra-operative fluoroscopic view showing completed procedure. A mechanically stable construct formed following judicious use of interfragmentary compression and locking screws.

high rates of infection and soft-tissue complications requiring multiple surgeries.

Preservation of soft-tissue integrity is particularly critical with distal tibial injuries because the antero-medial cortex is subcutaneous, and the remaining soft-tissue envelope is thin. As a result, minimally invasive plating techniques have been developed. LCP using MIPPO causes minimal soft tissue damage. Therefore, it has a biological advantage over ORIF in that it preserves the periosteal blood supply and as a result increases the chance of healing $[8,17]$.

Minimally invasive percutaneous plate osteosynthesis (MIPPO) is a well-documented technique that offers biological advantages and appears to be a reasonable treatment option for complex unstable distal tibial or pilon fractures. The literature is full of studies from developed nations $[8,10,16-18]$, but studies from developing nations are lacking. Aksekili et al. [10], Borg et al. [17], Redfern et al. [18] reported good results for MIPPO using closed, indirect reduction and contoured dynamic compression plates for distal tibial fractures $[10,17,18]$.

Infection rates vary between 0 and $50 \%$ subsequent to operative management of distal tibial fractures [17-21]. The highest rate of infection was reported with conventional ORIF [22]. Infection rates in the MIPPO technique are better than in previously reported ORIF studies and are comparable to external fixation technique $[17,20,21,23$ 27]. A solemn decline in the rate of infection, especially deep infection has been observed in patients managed with MIPPO technique. None of our patient developed deep infection, however two patient developed superficial infection, which is comparable to the existing literature.

Although, mal-reduction and mal-union has been reported in literature with minimally invasive approaches, but is of less of concern in comparison to intramedullary nailing and external fixator [2]. This is also reflected in our study with only $2(7.7 \%)$ patients having tibial mal-alignment of $5^{\circ}$ of valgus and none of the patient developing significant recurvatum or pro-curvatum post-operatively and at union, which is in conformation with Maffulli et al. [28] and Khoury et al. [29]. This can be further minimized by accurately pre-contouring the plate or using the pre-contoured plates, making a mechanically stable construct byusing the technique wisely and fixing associated fibular fracture. However, the clinical impact of fixing associated fibular fractures when stabilizing distal tibial fractures remains indefinite. In our experience, fibular plating is a useful adjunct in the setting of significant metaphyseal comminution in which rotational and sagittal alignment may be difficult to maintain with plate alone. We found that concurrent fibular fixation along with percutaneous plating is helpful in restoring length, alignment and indirect reduction of the tibial fracture in displaced and comminuted fractures.

Mean fracture healing was observed at 4.5 months (3.5-8 months). Our results were comparable to other studies using the MIPPO technique $[10,17,28,29]$. Comparing the results with other methods of fixation, our results are better in comparison with ORIF and external fixation [2-6,17,23]. However, Hasenboehler et al. [30] reported prolonged healing times with MIPPO technique in simple fracture patterns [30]. One patient in open fracture group developed delayed union. The fracture progressed to successful union at ten month postoperatively.

Distal tibial plating by MIPPO technique provides rigid fixation, which obscures the routine use of plaster immobilization in postoperative period. This in turn allows early active ankle exercises. In our study, mean AOFAS score of 88 was noted at 14.3 months of mean follow-up. Our study also favors that ankle stiffness is not a problem in management of distal tibial fractures with MIPPO technique $[17,28,29,31]$.

In terms of weight bearing, our results are comparable to Maffulli et al. [28], Khoury et al. [29], Bahari et al. [31], Redfern et al. [18] instructed full weight-bearing ambulation following clinical evaluation and the radiological appearance of callus formation [18]. Likewise in our study, non weight bearing ambulation was started on $3^{\text {rd }}$ postoperative day, progressing to partial weight bearing at 6 to 8 weeks (mean 6.2 weeks) and full weight bearing ambulation at 12 to 14 weeks (mean 12.4 weeks) at clinico-radiological evidence of union. Ozkoc and Tandonan RN [32] reported that bone failure can be prevented by using plates in a manner by distributing stresses over a large area [32]. In current study, due care was taken to follow above observation which was reflected in our results also, with no case of bone or implant failure.

As already discussed none of our case had hardware failure, but plate impingement was of significant concern in four (14.3\%) cases. None of our patient opted for hardware removal as plate impingement was not interfering with activities of daily living. Similar results were reported by Cheng et al. [33] whereby none of their cases also not opted for plate removal [33]. However a higher percentage of plate impingement was reported by Lau et al. [34] whereby $52 \%(25 / 48)$ of 
Citation: Asif N, Siddiqui YS, Jain JK, Zahid M, Abbas M, et al. (2014) Minimally Invasive Percutaneous Plate Osteosynthesis (Mippo) in Distal Tibial Fractures - A Prospective Study from Developing Nation. J Trauma Treat S2: 009. doi:10.4172/2167-1222.S2-009

distal tibial fracture cases treated by MIPPO had the implants removed due to skin impingement. Plate impingement resulting in skin irritation problems could be related to the thin subcutaneous nature of distal tibia and suboptimal pre-contouring of plates. Furthermore, the residual malreduction also contributes to the imprecision of plate and bone contouring. Anterior knee pain is not an issue with MIPPO technique in comparison to intramedullary nailing $[2,3]$.

One of the shortcomings of MIPPO is that it does not allow direct visualization of the fracture site and hence the operating surgeon is reliant on intra-operative fluoroscopy to confirm the reduction, plate insertion, insertion of drill guides and locking screws, thus increasing radiation exposures. To overcome the risk of radiation exposure, Rijal et al. [35] described a technique for minimizing radiation and incision with MIPPO (MRI-MIPPO) [35]. Author has used another plate of same length (plate over plate technique) for locating the locking holes with wires, so that all proximal and distal screws can be inserted with stab incision. In their study, 3-4 image intensifier views were required for completing the entire procedure.

To conclude, treatment of unstable distal tibial or pilon fractures remains challenging and is dictated by the fracture displacement, comminution, intra-articular extension and injury to the soft-tissue envelope. MIPPO is a well-documented technique that offers biological advantages and appears to be a reasonable treatment option for complex unstable distal tibial or pilon fractures that are not suited for intramedullary nailing. The literature is full of studies from developed nations, but studies from developing nations are lacking. Nevertheless small numbers of patients were involved in our study to draw any definitive conclusions, but our encouraging results will motivate the trauma surgeons from the developing nations to use MIPPO technique in managing distal tibial fractures, in an endeavor to reduce morbidity and associated complications, especially of the soft tissues.

\section{References}

1. Bedi A, Le TT, Karunakar MA (2006) Surgical treatment of nonarticular distal tibia fractures. J Am Acad Orthop Surg 14: 406-416.

2. Im GI, Tae SK (2005) Distal metaphyseal fractures of tibia: a prospective randomized trial of closed reduction and intramedullary nail versus open reduction and plate and screws fixation. J Trauma 59: 1219-1223.

3. Konrath G, Moed BR, Watson JT, Kaneshiro S, Karges DE, et al. (1997) Intramedullary nailing of unstable diaphyseal fractures of the tibia with distal intraarticular involvement. J Orthop Trauma 11: 200-205.

4. Marsh JL, Bonar S, Nepola JV, Decoster TA, Hurwitz SR (1995) Use of an articulated external fixator for fractures of the tibial plafond. J Bone Joint Surg Am 77: 1498-1509.

5. Mockford BJ, Ogonda L, Warnock D, Barr RJ, Andrews C (2003) The early management of severe tibial pilon fractures using a temporary ring fixator. Surgeon 1: 104-107.

6. Mosheiff R, Safran O, Segal D, Liebergall M (1999) The unreamed tibial nail in the treatment of distal metaphyseal fractures. Injury 30: 83-90.

7. Siebenrock KA, Schillig B, Jakob RP (1993) Treatment of complex tibial shaft fractures. Arguments for early secondary intramedullary nailing. Clin Orthop Relat Res: 269-274.

8. Farouk O, Krettek C, Miclau T, Schandelmaier P, Guy P, et al. (1997) Minimally invasive plate osteosynthesis and vascularity: preliminary results of a cadaver injection study. Injury 28 Suppl 1: A7-12.

9. Frigg R (2003) Development of the Locking Compression Plate. Injury 34 Supp 2: B6-10.

10. Aksekili MA, Celik I, Arslan AK, Kalkan T, UAYurlu M (2012) The results of minimally invasive percutaneous plate osteosynthesis (MIPPO) in distal and diaphyseal tibial fractures. Acta Orthop Traumatol Turc 46: 161-167.

11. Muller ME, Nazarian S, Koch P, Schatzker J (1990) The AO classification of fractures of long bones. Berlin: Springer.
12. Gustilo B, Anderson JT (1976) Prevention of infection in the treatment of one thousand and twenty-five open fractures of long bones. J Bone Joint Surg 58 453-458.

13. Pavolini B, Maritato M, Turelli L, D'Arienzo M (2000) The llizarov fixator in trauma: a 10-year experience. J Orthop Sci 5: 108-113.

14. Piper KJ, Won HY, Ellis AM (2005) Hybrid external fixation in complex tibial plateau and plafond fractures: an Australian audit of outcomes. Injury 36: 178184

15. Rammelt S, Endres T, Grass R, Zwipp H (2004) The role of external fixation in acute ankle trauma. Foot Ankle Clin 9: 455-474, vii-viii.

16. Borrelli J Jr, Prickett W, Song E, Becker D, Ricci W (2002) Extraosseous blood supply of the tibia and the effects of different plating techniques: a human cadaveric study. J Orthop Trauma 16: 691-695.

17. Borg T, Larsson S, Lindsjo U (2004) Percutaneous plating of distal tibial fractures. Preliminary results in 21 patients. Injury 35: 608-614.

18. Redfern DJ, Syed SU, Davies SJ (2004) Fractures of the distal tibia: minimally invasive plate osteosynthesis. Injury 35: 615-620.

19. Frigg R (2001) Locking Compression Plate (LCP). An osteosynthesis plate based on the Dynamic Compression Plate and the Point Contact Fixator (PCFix). Injury 32 Suppl 2: 63-66.

20. Bonar SK, Marsh JL (1994) Tibial Plafond Fractures: Changing Principles of Treatment. J Am Acad Orthop Surg 2: 297-305.

21. Blauth M, Bastian L, Krettek C, Knop C, Evans S (2001) Surgical options for the treatment of severe tibial pilon fractures: a study of three techniques. J Orthop Trauma 15: 153-160.

22. Teeny SM, Wiss DA (1993) Open reduction and internal fixation of tibial plafond fractures. Variables contributing to poor results and complications. Clin Orthop Relat Res : 108-117.

23. Perren SM (2001) Minimally invasive internal fixation history, essence and potential of a new approach. Injury 32 Suppl 1: SA1-3.

24. Sands A, Grujic L, Byck DC, Agel J, Benirschke S, et al. (1998) Clinical and functional outcomes of internal fixation of displaced pilon fractures. Clin Orthop Relat Res : 131-137

25. Sirkin M, Sanders R, DiPasquale T, Herscovici D (2004) A staged protocol for soft tissue management in the treatment of complex pilon fractures. J Orthop Trauma $13: 78-84$

26. Helfet DL, Shonnard PY, Levine D, Borrelli J Jr (1997) Minimally invasive plate osteosynthesis of distal fractures of the tibia. Injury 28 Suppl 1: A42-47.

27. Helfet DL, Koval K, Pappas J, Sanders RW, DiPasquale T (1994) Intraarticular "pilon" fracture of the tibia. Clin Orthop Relat Res : 221-228.

28. Maffulli N, Toms AD, McMurtie A, Oliva F (2004) Percutaneous plating of distal tibial fractures. Int Orthop 28: 159-162.

29. Khoury A, Liebergall M, London E, Mosheiff R (2002) Percutaneous plating of distal tibial fractures. Foot Ankle Int 23: 818-824.

30. Hasenboehler E, Rikli D, Babst R (2007) Locking compression plate with minimally invasive plate osteosynthesis in diaphyseal and distal tibial fracture: a retrospective study of 32 patients. Injury 38 : 365-370.

31. Bahari S, Lenehan B, Khan H, McElwain JP (2007) Minimally invasive percutaneous plate fixation of distal tibia fractures. Acta Orthop Belg 73: 635640 .

32. Ozkoç G, Tandonan RN (2006) Orthopaedic approach in osteoporetic fractures [Article in Turkish] TOTB<D Dergisi 5: 1-2.

33. Cheng W, Li Y, Manyi W (2011) Comparison study of two surgical options for distal tibia fracture-minimally invasive plate osteosynthesis vs. open reduction and internal fixation. Int Orthop (SICOT) 35: 737-742.

34. Lau TW, Leung F, Chan CF, Chow SP (2008) Wound complication of minimally invasive plate osteosynthesis in distal tibia fractures. Int Orthop 32: 697-703.

35. Rijal L, Sagar G, Mani K, Joshi KN, Joshi A (2013) Minimizing radiation and incision in minimally invasive percutaneous plate osteosynthesis (MIPPO) of distal tibial fractures. Eur J Orthop Surg Traumatol 23: 361-365. 